\title{
Zur Form und Struktur von Hypothesen im Rahmen der physiotherapeutischen Untersuchung am Beispiel der beobachtenden Ganganalyse
}

Qualitative Think-Aloud-Analyse

\section{On the Form and Structure of Hypotheses within Physiotherapy Using the Example of Observational Gait Analysis}

\author{
Qualitative Think Aloud Study
}

Autoren

Vivien Gers, Annette Irene Probst, Thomas Schöttker-Königer

Institut

HAWK Hochschule für angewandte Wissenschaft und Kunst, Fakultät Soziale Arbeit und Gesundheit, Hildesheim

Schlüsselwörter

Hypothesenbildung, hypothetisch-deduktives Denken, Clinical Reasoning, beobachtende Ganganalyse, Think Aloud

Key words

hypothesis develoment, hypothetic-deductive reasoning, clinical reasoning, observational gait analysis, think aloud

eingereicht 27.06.2019

akzeptiert 04.11.2019

Bibliografie

DOI https://doi.org/10.1055/a-1078-8700

Online-Publikation: 15.1.2020

physioscience 2020; 16: 53-60

(C) Georg Thieme Verlag KG, Stuttgart · New York

ISSN 1860-3092

Korrespondenzadresse

Vivien Gers, MSC PT

HAWK Hochschule für angewandte Wissenschaft und Kunst

Goschentor 1, 31134 Hildesheim

vivien.gers2@hawk.de

\section{ZUSAMMENFASSUNG}

Hintergrund Obwohl Hypothesen als zentraler Bestandteil des Clinical Reasonings in der Physiotherapie eine Grundlage für therapeutisches Handeln darstellen, gibt es in der physiotherapeutischen Fachliteratur wenige Anhaltspunkte für deren Bildung und deren Merkmale.

Ziel Das Ziel dieser Arbeit ist es, die Hypothesenbildung am Beispiel der beobachtenden Ganganalyse zu untersuchen. Dabei wird die Frage geklärt, inwieweit Hypothesen im wissenschaftlichen Sinn gebildet werden können. Dafür werden die Begriffe Hypothese und Heuristik differenziert erläutert.
Methode Zur Untersuchung der Hypothesenbildung wurde ein qualitativer Forschungszugang gewählt. Dabei wurden acht Experten aus dem Feld der Physiotherapie anhand der Think-Aloud-Methode bei der beobachtenden Ganganalyse untersucht.

Ergebnisse Es konnten drei unterschiedliche Hypothesentypen herausgearbeitet werden. Dabei handelt es sich um (1) zu bewertende, (2) zu interpretierende und (3) zu bewertende und konditionale Annahmen. Keine der von den Experten gebildeten Hypothesen entspricht den Kriterien, die an Hypothesen im wissenschaftlichen Sinn heranreichen. Die Hypothesentypen entsprechen eher der Form, die wissenschaftstheoretisch Heuristiken zugeordnet werden.

Schlussfolgerungen Für die beobachtende Ganganalyse zeigt sich ein hypothesengeleitetes Vorgehen, welches an Heuristiken orientiert ist. Der Begriff der Hypothesenbildung wird derzeit in der Physiotherapie ungenau benutzt und sollte weiter untersucht werden, um den Wert von Annahmen, Heuristiken und echten Hypothesen für den Prozess der therapeutischen Entscheidungsfindung zu präzisieren und in Lehre und Forschung umsetzbar zu machen.

\section{ABSTRACT}

Background Hypotheses represent a fundamental part of the clinical reasoning process and thus are an essential part of the therapeutic work of any physiotherapist. Nonetheless, there is only very limited research available that seeks to define or characterize different forms of hypotheses within the physiotherapy field.

Objective The primary goal of this study is to investigate how hypotheses are generated using the example of observational gait analysis. The question is clarified to what extent hypotheses are formed in a scientific sense. For this purpose, the differentiation of the terms hypothesis and heuristic is undertaken.

Method Drawing on a qualitative research approach, Think Aloud surveys with a total of eight physiotherapists who have advanced training in terms of gait analysis were conducted. 
Results As a result, three types of hypotheses are proposed, namely (1) evaluative, (2) interpretative and (3) evaluative and conditional hypotheses. Surprisingly, none of the types formed by the experts attended in the Think Alouds corresponds to the criteria that come close to hypotheses in a scientific sense. The identified types of hypotheses correspond more to the form that is assigned to heuristics in scientific theory.
Conclusion Overall, the physiotherapeutic gait analysis follows a hypothesis-guided procedure based on heuristics. The present concept of hypothesis formation is currently used rather imprecisely in physiotherapy and should be further investigated in order to specify the value of assumptions, heuristics and real hypotheses for the process of therapeutic decision-making and to make them applicable in teaching and research.

\section{Einleitung}

In der Physiotherapie wird davon ausgegangen, dass die Hypothesenbildung eine Grundlage für physiotherapeutisches Handeln ist [1-3]. Nach dem Prinzip des hypothetisch-deduktiven Reasonings werden innerhalb der Untersuchung von Patienten verschiedene Daten und Informationen erfasst, um in einem weiteren Schritt darauf aufbauend erste Hypothesen zu generieren [2]. Auf Basis dessen, was ein Patient selbst an Vorerfahrungen und Hypothesen angibt, werden Hypothesen entwickelt, bis es zur Diagnosestellung und anschließenden Behandlung kommt. Der weitere Verlauf des Untersuchungsprozesses wird als „collaborative process between physiotherapists an patients“ beschrieben [2]. Neben einem hypothetisch-deduktiven Vorgehen wird auch einem interpretativen, narrativ orientierten Vorgehen unter Einbezug des Patienten eine Rolle eingeräumt [2]. Die Bildung von Hypothesen im Untersuchungsprozess wurde bereits für Handlungsfelder wie z. B. der Neurologie, Orthopädie und mit verschiedener Klientel untersucht [2-4]. Diese Studien bestätigen das Grundmodell des hypothetisch-deduktiven Reasonings und spezifizieren einzelne Schritte der Hypothesenbildung, die von Physiotherapeuten vorgenommen werden (Hypothesenformulierung, Prüfung, Bewertung) [2-4]. Hypothesen werden zudem in sogenannte Hypothesenkategorien sortiert, um das Wissen zu strukturieren [1, 5]. Hypothesenkategorien sind z. B. Ursache der Symptome, Aktivität und Partizipation oder beitragende Faktoren zu einem Problem $[1,5]$. Das heißt, dass zunächst jede Information und Wahrnehmung des Physiotherapeuten als Hypothese kategorisiert werden kann. Dabei werden keine festen Definitionen oder Kriterien für die Form von Hypothesen benannt. Eine in der physiotherapeutischen Untersuchung formulierte Hypothese lautet z. B. „mechanische Ursache des Problems“ und könnte in die Hypothesenkategorie „Ursache der Symptome“ einsortiert werden [3, 6]. Die Formulierung steht zunächst als Nebensatz für sich, für die Form oder inhaltliche Reichweite der Hypothese gibt es keine konkreten Angaben.

\section{Begriffserläuterung}

Der Begriff Hypothese nimmt in der empirischen Forschung die Form einer Annahme an, die eine Aussage über Erkenntnisgegenstände in Form von Sätzen ausdrückt [7]. In einem positivistischen Erkenntnisparadigma werden Hypothesen zu Beginn eines Forschungsprozesses aufgestellt und im Rahmen eines deduktiven Vorgehens überprüft [8]. Angelehnt an ein interpretatives Erkenntnisparadigma sind Hypothesen nicht vorab formuliert, sondern resultieren am Ende eines Forschungsprozesses im Sinne von neuen Erkenntnissen [8]. Bei wissenschaftlichen Hypothesen handelt es sich vorwiegend um Zusammenhangshypothesen, welche in diverse Untergruppen sortiert werden können [9-11]. Darüber hinaus werden bestimmte Kriterien wie Logik und Widerspruchsfreiheit, eine bestimmte Formstruktur (oftmals die eines Konditionalsatzes), Überprüfbarkeit sowie Falsifizierbarkeit für Hypothesen formuliert [9, 10].

Im Zuge eines interpretativen Erkenntnisparadigmas spielen anstelle von Hypothesen Heuristiken zu Beginn des Forschungsprozesses eine Rolle $[12,13]$. Heuristiken ermöglichen es, das Vorwissen zu strukturieren und den Gegenstandsbereich zu bestimmen [12, 13]. Der Begriff Heuristik wird definitorisch in der Psychologie als Vorgehensweise bei der Lösung von Problemen bzw. als Erfindungskunst und Anleitung zum Gewinn neuer Erkenntnisse beschrieben [14]. So wird ein bestimmter Bereich vereinfacht und der Erkenntnisgewinn ermöglicht [15]. Dafür wird nicht mit fest definierten Hypothesen gearbeitet, sondern mit Vorannahmen und Generalisierungen [15]. Heuristiken werden ebenfalls als Vorform einer Hypothese beschrieben [9]. Als „Hilfskonstruktion“ [9] zeigt eine Heuristik auf, dass in einer Annahme ein Zusammenhang zwischen zwei Variablen besteht, welcher jedoch noch nicht genau bestimmt ist (z. B., dass Ursache und Folge noch nicht festgelegt sind) [9].

\section{Hypothesenbildung in der beobachtenden Ganganalyse}

Für die Untersuchung der Hypothesenbildung in der Physiotherapie ist neben den bereits untersuchten Handlungsfeldern die beobachtende Ganganalyse ein weiteres mögliches Feld. Die Ganganalyse ist ein Untersuchungsinstrument, bei dem auf Grundlage von Beobachtungen anhand spezifischer Gangkriterien die Identifikation von strukturellen und aktivitätsbedingten Limitationen vorgenommen wird und die Interventionsplanung sowie Effektivitätsprüfung der Intervention folgt [16-19]. Es ist davon auszugehen, dass im Kontext des hypothetisch-deduktiven Reasonings als Grundlage für therapeutisches Handeln auch während der beobachtenden Ganganalyse Hypothesen gebildet werden. Die Hypothesenbildung bei der beobachtenden Ganganalyse wird jedoch nur in wenigen Studien beleuchtet [20]. In den Studien von Watelain et al. [20] wurden Ganganalysestrategien von Physiotherapeuten auf Basis der verwendeten Gangkriterien erforscht. Dabei wurden unter Anwendung eines qualitativen Forschungszuganges Kategorien gebildet, die in „beschreibenden“ oder „interpretativen“ Ganganalysestrategien resultieren, allerdings ohne Hypothesen im Rahmen dieser Strategien zu definieren [20]. 
Das Ziel der Forschungsarbeit ist es, den Prozess der Hypothesenbildung am Beispiel der beobachtenden Ganganalyse zu untersuchen. Die Hypothesenbildung ist für die Formulierung einer physiotherapeutischen Diagnose grundlegend. Daher wird die Frage geklärt, inwieweit Hypothesen am Beispiel der beobachtenden Ganganalyse Hypothesen im wissenschaftlichen Sinn entsprechen und ob sie von Heuristiken abzugrenzen sind. Die Ergebnisse können im herausfordernden Prozess des Interpretierens von Beobachtungen für Physiotherapeuten von Nutzen sein und ein präziseres Handeln im Hinblick auf weitere Untersuchungsschritte anleiten. Diese Arbeit behandelt dabei nicht den Abgleich bzw. die Einordnung von Clinical Reasoning mit Problemlösemodellen oder Handlungstheorien, sondern zieht als Grundlage das hypothetisch-deduktive Reasoning heran.

\section{Methode}

Um das Forschungsziel zu erreichen, wird der qualitative Forschungsansatz Think Aloud (TA) genutzt und der Beobachtungsprozess während der beobachtenden Ganganalyse auf einer ablauforientierten und formalen Ebene erfasst. Die ablauforientierte Ebene betrifft die einzelnen Phasen der Beobachtung und adressiert Forschungsfrage 1: Welche Vorgehensweisen wenden Physiotherapeuten innerhalb der beobachtenden Ganganalyse an? Die formale Ebene bezieht sich auf die Beschreibungsweise der Beobachtungen und die gebildeten Hypothesen. Die Forschungsfrage 2 lautet: Welche Formen von Hypothesen entstehen während der beobachtenden Ganganalyse?

Die empirischen Daten für die vorliegende Forschungsarbeit wurden mit der Think-Aloud-Methode (Methode des Lauten Denkens) erhoben. Das grundsätzliche Ziel ist, Vorgänge des menschlichen Bewusstseins zu externalisieren indem alle Gedanken, die beim Bearbeiten einer Problemstellung oder Lösen einer Fragestellung entstehen, verbalisiert werden [21, 22]. In verschiedenen Studien hat Think Aloud bereits Anwendung gefunden, um Gedanken- und Entscheidungsprozesse von Physiotherapeuten während der Untersuchung und Behandlung abzubilden [20, 2325]. Dabei konnte durch die Think-Aloud-Methode ein hypothetisch-deduktives Reasoning durch die Bildung von Hypothesen generell bestätigt [23-25] werden. Es zeigte sich, dass bei der Beobachtung von Patienten bestimmte Analysestrategien verwendet werden [20]. Da diese Studien unter Anwendung der Methode anschauliche Ergebnisse erbrachten, befürworten diese die Anwendung von Think Aloud für die vorliegende Forschungsarbeit. Die ethischen Grundsätze der aktuellen Fassung der Deklaration von Helsinki wurden dabei berücksichtigt.

\section{Samplebeschreibung und Datenerhebung}

Für die Datenerhebung standen acht Physiotherapeuten zur Verfügung. Sechs Physiotherapeuten hatten Fortbildungen nach dem Funktionelle-Bewegungslehre-Konzept (FBL) nach KleinVogelbach, zwei Physiotherapeuten Fortbildungen im Gehen-Verstehen-Konzept nach Götz-Neumann bzw. in der Ganganalyse nach Jacqueline Perry absolviert [16, 27]. Die ausgewählten Physiotherapeuten sind aufgrund ihrer Fortbildungen und Berufserfahrung Experten in der Ganganalyse und daher für die
- Tab. 1 Übersicht des Samples: Physiotherapeuten 1-8 mit Angaben zu Berufserfahrung, Ganganalysefortbildung und Dauer Think Aloud.

\begin{tabular}{|l|l|l|l|}
\hline ID & $\begin{array}{l}\text { Berufserfahrung } \\
\text { [Jahre] }\end{array}$ & $\begin{array}{l}\text { Ganganalyse- } \\
\text { fortbildung }\end{array}$ & $\begin{array}{l}\text { Dauer Think } \\
\text { Aloud } \\
\text { [Minuten] }\end{array}$ \\
\hline P1 & 28 & FBL & 32 \\
\hline P2 & 45 & FBL & 20 \\
\hline P3 & 35 & FBL & 32 \\
\hline P4 & 32 & FBL & 28 \\
\hline P5 & 30 & FBL & 55 \\
\hline P6 & 37 & FBL & 39 \\
\hline P7 & 36 & $\begin{array}{l}\text { Ganganalyse nach } \\
\text { Perry [26] }\end{array}$ & 20 \\
\hline P8 & 18 & $\begin{array}{l}\text { Gehen-Verstehen- } \\
\text { Konzept nach } \\
\text { Götz-Neumann [16] }\end{array}$ & 97 \\
\hline & & & \\
\hline FBL = Funktionelle Bewegungslehre nach Klein-Vogelbach, \\
\hline P= Physiotherapeut.
\end{tabular}

Forschungsarbeit besonders geeignet. Eine Übersicht zum Sample bietet $>$ Tab. 1.

Die Datenerhebung gliederte sich in die Phasen Vorbereitung, Aufwärmübung, Ganganalyse und Nachbesprechung. Im Vorfeld wurden zur Erprobung der Datenerhebung zwei Pilottestungen durchgeführt und ein Leitfaden erstellt, der nach den Testungen angepasst wurde. Die Teilnehmenden wurden über den Ablauf und die Methode informiert und gaben eine informierte Einwilligung zur Teilnahme. Daraufhin wurde die Beobachtung eines neutralen Videos vorgenommen. Die Ganganalyse wurde anhand von zwei Gangvideos von Patienten, die Beschwerden beim Gehen vorweisen, durchgeführt. Die Aufgabe der Physiotherapeuten bestand in der Analyse der Videos und lautete: „Führen Sie einen Befund durch“. Während der Videoanalyse wurden die Physiotherapeuten aufgefordert, laut zu denken. Das gesprochene Wort wurde digital aufgezeichnet. In der Nachbesprechung wurden vertiefende Fragen über empfundene Herausforderungen und weitere benötigte Informationen gestellt.

\section{Datenauswertung}

Innerhalb der Datenauswertung wurde das gesprochene Wort transkribiert und mithilfe des Computerprogrammes $\mathrm{f} 4$ in ein verbales Protokoll überführt [21, 28]. Daraufhin wurden die Segmentierung und Kodierung des Think-Aloud-Protokolls vorgenommen. Innerhalb der Segmentierung fand die Unterteilung des Protokolls in Abschnitte statt. Daraufhin erfolgte das Kodieren, angelehnt an die Grounded Theory [29]. Nach der Grounded Theory wird aus dem qualitativen Datenmaterial eine Theorie gebildet, wobei die Auswertung in Verbindung mit dem Kontextwissen des Forschers und basierend auf Kodierungen stattfindet [30]. In der vorliegenden Forschungsarbeit gliederte sich die 


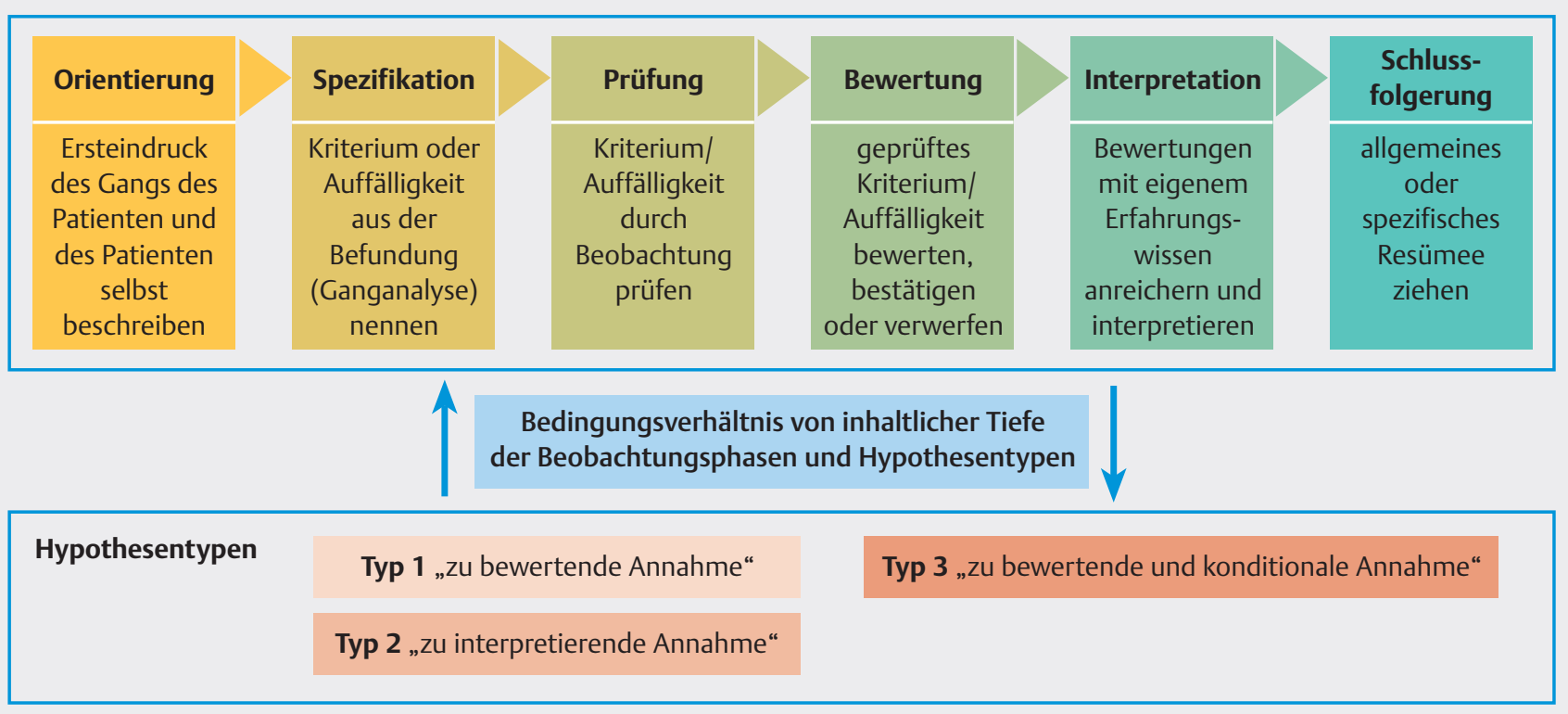

- Abb.1 Zusammenhang von Beobachtungsprozess und Hypothesentypen während der beobachtenden Ganganalyse. (Quelle: V. Gers; graf. Umsetzung: Thieme Gruppe)

Datenauswertung in drei Schritte und zielte insgesamt auf eine induktive Kategorienbildung bzw. Phasenbestimmung ab. In Schritt (1) wurde das offene Kodieren, indem alle Konzepte bzw. Vokabeln identifiziert wurden, vorgenommen [29]. Die Konzepte bzw. Vokabeln repräsentieren Phänomene, die in der Analyseeinheit vorhanden sind. Auf die Forschungsarbeit bezogen wurden in diesem Schritt Kriterien, die von den Physiotherapeuten bei der Ganganalyse herangezogen wurden, beschrieben und kategorisiert. In Schritt (2) erfolgte durch den Forscher die Rekonstruktion von Beziehungen und Verbindungen zwischen den gefundenen Konzepten [28]. Auf das Datenmaterial bezogen wurden im Rahmen der Verbindung der Konzepte die Beobachtungsprozesse der acht Physiotherapeuten auf einer ablauforientierten Ebene konstatiert. Die ablauforientierte Ebene umfasst die einzelnen Phasen der Beobachtung. Die Einteilung in diese Phasen wurde durch sprachliche Übergänge und inhaltliche Überschneidungen bzw. Unterschiede vorgenommen. Das Datenmaterial wurde dahingehend untersucht, ob sich individuelle oder kollektive Vorgehensweisen der Physiotherapeuten während der Beobachtung zeigten. Abschließend folgte in Schritt (3), der Auswertung, die Beschreibung übergeordneter Gedankenprozesse [28]. Die Gedankenprozesse der untersuchten Physiotherapeuten wurden in diesem Analyseschritt in Form von generierten Hypothesen auf formaler Ebene beschrieben.

\section{Ergebnisse}

Zur Strukturierung wird zunächst der Beobachtungsprozess der Ganganalyse, der basierend auf den Think-Aloud-Protokollen extrahiert wurde und als Orientierung für die Einordnung der Hypothesen dient, auf der ablauforientierten Ebene beschrieben. Anschließend folgt die Typisierung der Hypothesen auf formaler Ebene.

\section{Beobachtungsprozess der Physiotherapeuten}

Der Beobachtungsprozess aller untersuchten Physiotherapeuten gliederte sich in sechs Beobachtungsphasen ( $\bullet$ Abb. 1). Die erste Phase ist die „Orientierung“, in der sich die Physiotherapeuten in die Beobachtungssituation einfinden. Die Einteilung in diese Phase erfolgt zunächst auf inhaltlicher Ebene. Ein- und Ausstieg in bzw. aus dieser Phase werden darüber hinaus sprachlich markiert. Die Probanden sprachen teilweise selbst von einem „ersten Eindruck“ oder „zuerst das Gesamtbild“ (z. B. P3, P4). Darauf folgt die Phase der „Spezifikation“, in der die Kriterien der Ganganalyse oder Auffälligkeiten im Rahmen der Beobachtung genannt werden. Die Phase der Spezifikation ist durch sprachliche Marker wie „jetzt“ oder „also“ und durch das Nennen eines Kriteriums markiert (P1, P8). Inhaltlich beziehen sich die Gangkriterien und Auffälligkeiten z. B. auf Gangphasen, Gelenkstellungen oder mögliche Beschwerden des Patienten beim Gehen.

In der nächsten Beobachtungsphase „Prüfung“ werden die Gangkriterien oder Auffälligkeiten durch wiederholte, genaue Beobachtung oder das Abspielen des Videos in verlangsamter Geschwindigkeit geprüft und mit bestimmten Definitionen oder Erwartungswerten abgeglichen. Sprachliche Marker, die diese Phase markieren, sind z. B. „da muss ich noch ein bisschen genauer schauen“ oder „jetzt habe ich es nochmals kontrolliert“ (P3, P8). Ziel dieser Phase ist, zu einer Bewertung des Gangkriteriums oder der Auffälligkeit zu gelangen. Nach einer anfänglichen Spezifikation („Ich finde, dass er vielleicht wenig Dorsalextension in den Zehen zeigt“ (P8)) kann bei Probanden bereits eine Prüfung erfolgen (,Ich gucke mir den nächsten Schritt noch einmal an, ob man das da besser sieht“ (P8)). Die Probanden denken über die beobachteten Auffälligkeiten genauer nach und bestätigten bzw. verwerfen ihre Annahmen als Ergebnis dieser Prüfung. Daher ist die „Prüfung“ eng mit der Phase „Bewertung“ verknüpft. 
> Tab. 2 Typen von Hypothesen.

\begin{tabular}{|l|l|l|l|}
\hline & Typ 1: zu bewertende Annahme & Typ 2: zu interpretierende Annahme & $\begin{array}{l}\text { Typ 3: zu bewertende und konditionale } \\
\text { Annahme }\end{array}$ \\
\hline Inhalt & $\begin{array}{l}\text { Bewertung einer vorausgehenden } \\
\text { Annahme }\end{array}$ & $\begin{array}{l}\text { über die Beobachtung hinausgehende } \\
\text { Annahme }\end{array}$ & $\begin{array}{l}\text { Bedingungszusammenhang zweier oder } \\
\text { mehrerer Annahmen, Begründung }\end{array}$ \\
\hline Form, Struktur & Satz im Indikativ & Satz im Konjunktiv & Finalsatz/Konditionalsatz \\
\hline Beispiele & $\begin{array}{l}\text { „Der Übergang von Mid Stance } \\
\text { zum Terminal Stance auf dem } \\
\text { rechten Bein findet bei der } \\
\text { Probandin verkürzt statt“ (P7). }\end{array}$ & $\begin{array}{l}\text { „Die Muskulatur in der Wade könnte in } \\
\text { ihrer Dehnfähigkeit vermindert sein“ (P8). }\end{array}$ & „Der Proband weist einen größeren Arm- \\
pendel auf, um das Bein zu entlasten“ (P 1).
\end{tabular}

In der Phase „Bewertung“ wird das Gangkriterium bzw. die Auffälligkeit durch die Hinzunahme von Adjektiven oder Adverbien bewertet: „Er hat hier eine relativ gute Zehenextension“ (P8). Es ist möglich, dass Probanden in dieser Phase bereits Sicherheit in ihren Bewertungen erlangen und eine Beobachtung bestätigen oder verwerfen. Wenn sie noch unsicher sind, durchlaufen sie in einer iterativen Schleife die Phasen „Spezifizieren“ oder „Prüfung".

Die folgende Phase „Interpretation“ beinhaltet, dass weiterführende Annahmen zu Beobachtungen und Bewertungen formuliert und mit Erfahrungswissen angereichert werden. Die Annahmen gehen dabei über die reine Beobachtung hinaus und können verschiedene Bereiche wie z. B. Ursachen für Abweichungen, Problemorte oder Beschwerden adressieren. Proband P7 interpretiert z. B. die Annahme eines erhöhten Tonus im Schulterbereich als mögliche Folge oder Ursache eines auffälligen Ganges so: „Was [...] auf einen Hypertonus im Schulterbereich schließt“ (P7). Eine Annahme lässt sich jedoch allein durch Beobachtung nicht prüfen oder bestätigen.

Zum Abschluss der Beobachtung wird eine beobachtungsbezogene Schlussfolgerung vorgenommen. Diese ist entweder ein allgemeines oder spezifisches Resümee der gesammelten Informationen auf Basis aller Beobachtungsdaten. Sprachlich zeigen sich diese Resümees z. B. durch Aussagen wie „ich komme zu dem Resultat“ oder „die Hauptabweichung ist“ (P1, P8). Wenn die Probanden in der Abschlussphase der Beobachtung kein Resümee vornehmen können, ist eine erneute Iteration des Beobachtungsprozesses möglich.

\section{Hypothesentypen: Formen von Annahmen während der Hypothesenbildung}

Innerhalb des Beobachtungsprozesses lassen sich drei verschiedene Formen von Annahmen ableiten, die als Hypothesentypen in - Tab. 2 dargestellt werden. Eine Hypothese kann grundsätzlich als Annahme verstanden werden, die in unterschiedlichen Phasen des Beobachtungsprozesses, mit unterschiedlichem Inhalt, in einer unterschiedlichen Form und mit unterschiedlichem Generalisierungsgrad formuliert wird. Die Hypothesenbildung ist durch die Aktivitäten Spezifizieren, Prüfen, Bewerten, Interpretieren und Schlussfolgern geprägt.

Typ 1 (zu bewertende Annahme) zeichnet sich durch eine Aussage im Indikativ aus, die eine Wertung bzw. eine Beurteilung aufgrund der vorgenommenen Beobachtung beinhaltet. Diese Annahme kann durch einen oder mehrere Prüfvorgänge bestätigt oder widerlegt werden. Zum Beispiel wird vermutet, dass die Spurbreite des Patienten auffällig ist: „Ich würde sagen, dass die Spur rechts verbreitert ist, etwas mehr von der rechten Seite wie von der linken“ (P5). Nach einem weiteren Prüfvorgang wird diese Annahme als Bewertung bestätigt und gilt als zu bewertende Annahme: „Ja, er geht mit dem rechten Fuß ein bisschen mehr zur Seite“ (P5). Bei diesem Beispiel wird eine Auffälligkeit spezifisch benannt und bewertet, es ist vorstellbar, dass der Proband diese im weiteren Untersuchungsprozess weiterverfolgen wird.

Typ 2 (zu interpretierende Annahme) zeichnet sich durch weiterführende Interpretationen aus und wird durch das Verknüpfen von Informationen und Heranziehen von zusätzlichem Wissen geprägt. Dabei wird die Form eines Konjunktivsatzes gewählt. Dieser formuliert bestimmte Zusammenhänge oder Ursachen als Mutmaßungen. Am Beispiel „Der Proband könnte Probleme oder Beschwerden mit der Schulter haben“ (P6) wird deutlich, dass die Interpretation über die Beobachtung hinausgeht. Es handelt sich eher um eine generalisierte Annahme über einen bestimmten Aspekt, die im weiteren Untersuchungsprozess überprüft werden müsste.

Typ 3 (zu bewertende und konditionale Annahme) ist in einen komplexeren Satz gebettet. Dieser zeigt einen hergestellten Zusammenhang zwischen zwei oder mehreren Aspekten, die in einem Bedingungs- bzw. Begründungsverhältnis stehen. Ein Beispiel: „Der Proband weist einen größeren Armpendel auf, um das Bein zu entlasten“ (P1). Typ 3 ist durch Konjunktionen wie „weil“ und „um“ oder sprachliche Markierungen wie „dazu führen“ oder „bedingen“ gekennzeichnet und bildet einen Finalsatz bzw. Kon- 
ditionalsatz. Dabei wird ein Zusammenhang zwischen zwei spezifischen Aspekten, die in einem Bedingungsverhältnis stehen oder deren Beziehung (z. B. Ursache und Folge) noch nicht genau zugeordnet ist, bestimmt.

Insgesamt zeigen Annahmen als Implikation einen weiteren Handlungsbedarf an. Durch weitere Methoden und Untersuchungen müssten sie überprüft und weiterentwickelt werden, damit Physiotherapeuten sie annehmen oder verwerfen können.

Die drei Typen von Hypothesen werden in den Phasen des Beobachtungsprozesses gebildet und sind in $\mathbf{A}$ Ab. 1 im Zusammenhang mit diesem dargestellt. Typ 3 ist in seiner formalen Struktur am komplexesten und wird durch die Phasen Prüfung, Bewertung und Interpretation gebildet. Er ist dadurch gekennzeichnet, dass Physiotherapeuten die genannten Phasen präzise und inhaltlich ausgedehnt vornehmen. Typ 1 und Typ 2 sind in ihrer formalen Struktur weniger komplex sind in der Regel dadurch gekennzeichnet, dass Physiotherapeuten die einzelnen Phasen im Beobachtungsprozess mit geringer inhaltlicher Tiefe oder weniger ausgedehnt als bei Typ 3 gestalten. Bezogen auf die Generalisierung bzw. Spezifizierung der verschiedenen Annahmen können sowohl spezifischere Zusammenhänge, die bereits in Bedingungszusammenhängen stehen, als auch generalisierte Annahmen, die als erste Orientierung dienen und in weiteren Untersuchungen spezifiziert werden müssen, formuliert werden. Auf diese Weise entsteht eine sich bedingende Beziehung der einzelnen Phasen des Beobachtungsprozesses mit der Hypothesenbildung. Die Beziehung ergibt sich einerseits durch die inhaltliche Tiefe sowie die Präzision, mit der die einzelnen Phasen im Beobachtungsprozess durchlaufen werden, wodurch unterschiedliche Hypothesentypen gebildet werden. Andererseits können formulierte Hypothesen einzelne Phasen des Beobachtungsprozesses und somit die Hypothesenbildung anregen, indem z. B. erneute Prüfvorgänge oder Spezifikationen vorgenommen werden.

\section{Diskussion}

Die vorliegende Forschungsarbeit hatte zum Ziel, die Hypothesenbildung von Physiotherapeuten, die eine beobachtende Ganganalyse durchführen, auf einer ablauforientierten und formalen Ebene zu erfassen. Angelehnt an Edwards und Jones [1, 2] wurde die Begrifflichkeit „Hypothese“ aufgenommen und verwendet, jedoch differenziert betrachtet.

\section{Methode und Limitationen des Studiendesigns}

Das Think Aloud erwies sich als geeignete Methode zur Extrahierung von Gedankenprozessen. Als Herausforderung zeigte sich, dass ein Gelingen von der Fähigkeit des Physiotherapeuten abhängig ist, tatsächlich laut zu denken. Zentral hierfür ist, dass die Gedanken ungefiltert im Moment des Denkens ausgesprochen und nicht als sprachliche Reflexion geäußert werden. Zieht man als Grundlage für das Funktionieren des lauten Aussprechens von Gedanken während einer Aufgabenausführung die Systematik von Wissensformen nach Polanyi heran, kann davon ausgegangen werden, dass für die Aufgabenstellung der Bewegungsanalyse anwendbares, praktisches Wissen benötigt wird [31]. Wenn der
Beobachtungs- und Analyseprozess intuitiv abläuft, können einzelne Schritte oder Strategien, obwohl das Wissen präsent ist, nicht zwingend verbalisiert werden und bleiben implizit [31]. Dies stellt eine Limitation der Methode dar. Eine weitere Limitation ergibt sich aus dem künstlichen Umfeld. Die Physiotherapeuten sollten anhand eines Videos und ohne die Patienten vorher zu kennen den Befund vornehmen. Dieses Setting stellt einen reduzierten Rahmen dar, in dem keine vollumfängliche Befundung und Diagnosestellung möglich ist. Das Setting wurde bewusst gewählt, um sich durch Reduzierung der Komplexität anzunähern. Wenn Physiotherapeuten Schwierigkeiten mit der Methode des lauten Denkens haben oder normalerweise mit anderen Patienten arbeiten als in den Videos gezeigten, besteht die Möglichkeit, dass Think Alouds zeitlich kürzer ausfallen.

\section{Hypothesen von Physiotherapeuten beim Clinical Reasoning}

Die beschriebenen Hypothesentypen sind denen von Jones beschriebenen initialen, globalen Hypothesen, die im Untersuchungsprozess von Physiotherapeuten gebildet werden [1, 5], ähnlich. Der Schritt der Hypothesenbildung kann durch die Ergebnisse dieser Forschungsarbeit differenziert werden. Hypothesen in Form von Annahmen werden schon während des Sammelns von Informationen in mehreren Iterationen geprüft und angepasst. Am Ende eines Untersuchungsschrittes (z. B. der beobachtenden Ganganalyse) kommt es zu beobachtungsbasierten Annahmen, die sich in einfachen, aber auch komplexen Zusammenhängen zeigen. Nach Edwards und Jones werden innerhalb des hypothetisch-deduktiven Vorgehens zunächst Hypothesen entwickelt und diese in einem nächsten Schritt durch Hinzunahme von weiteren Informationen (welche durch weitere, sich anschließende Untersuchungsmethoden generiert werden) evaluiert [2, 32]. Als Differenzierung des Schrittes der Hypothesenbildung nach Edwards und Jones werden Hypothesen, wie diese Forschungsarbeit aufzeigt, in iterativen Schritten schon innerhalb der Beobachtung selbst überprüft, angepasst, bestätigt und verworfen. Dieser Prozess geschieht, ohne dass neue Informationen z. B. durch andere Untersuchungsmethoden hinzukommen.

\section{Bearbeitungsstände von Hypothesen}

Die Typen von Hypothesen, die das Sample zeigt, sind den Bearbeitungsständen von Hypothesen im Wissenschaftsverständnis nach Häder ähnlich [10]. Es gibt demnach zu Beginn eines Arbeitsprozesses anstelle von Hypothesen eher Vermutungen, die noch keine Überprüfung erfahren haben [10]. Nach einem Prüfprozess werden Hypothesen als Prüfhypothesen bezeichnet [10]. Nach der Prüfung kann eine Hypothese eine bestätigte oder widerlegte Hypothese sein [10]. Die vorliegende Forschungsarbeit zeigt ebenfalls Annahmen auf, die durch Prüfungsvorgänge eine Bewertung erhalten und angenommen oder verworfen werden. In Anlehnung an die Systematik von Häder muss eine Annahme überprüfbar sein und aus ihrer Prüfung eine Bestätigung oder Widerlegung resultieren, damit Annahmen auch als Hypothese bezeichnet werden können. 


\section{Hypothesen in Abgrenzung zu Heuristiken}

Einerseits werden bei den Hypothesentypen von den Physiotherapeuten Zusammenhänge zwischen Aspekten formuliert, die in einem Konditionalsatz stehen und auf diese Weise Hypothesen in der empirischen Forschung ähneln. Andererseits werden Annahmen über Aspekte geäußert, die eher zur Orientierung dienen und zunächst in einem generalisierten Rahmen stehen, der die Möglichkeit von Prüfung und Weiterverfolgung offenlässt. Daher sind in diesem Stadium der Untersuchung vermehrt Heuristiken zu finden. Es handelt sich noch nicht um echte Hypothesen im wissenschaftlichen Sinn.

Wenn Hypothesentyp 3 mit der von Bortz und Döring beschriebenen „Wenn-dann-Heuristik“ verglichen wird [9], ergibt sich folgende Parallele. Hier werden Bedingungszusammenhänge von mehreren Aspekten formuliert. Es gibt also einen Zusammenhang, gleichzeitig bleibt offen, welcher Aspekt als Ursache und welcher Aspekt als Folge betrachtet wird. Ein Physiotherapeut formuliert z. B.: „Die Besonderheit ist am Fuß, dass er eigentlich nach außen geht [...]. Und meine Vermutung zumindest ist, dass das dazu führt, dass auch der Oberschenkel nach außen rotiert wird, was aber auch, wie gesagt andersherum eingeleitet werden könnte. (Unv.), das kann ich noch nicht ganz einordnen“ (P8). Der Zusammenhang der beiden Aspekte erscheint logisch und sinnvoll und stellt eine Kausalhypothese dar. Es ist aber ebenfalls möglich, dass die Drehung des Oberschenkels eine Folge der Stellung des Fußes (Ursache) ist. Dieser Annahme hat eher den Charakter einer Heuristik als einer Hypothese, da die Teile des Konditionalsatzes austauschbar sind [9]. Solange diese austauschbar sind, stellen sie im strengeren Sinn keine Hypothese dar. Daher verdichten sich im Rahmen dieser Forschungsarbeit die Hinweise, dass innerhalb des physiotherapeutisch hypothetisch-deduktiven Reasonings im Anfangsstadium einer Untersuchung eher Heuristiken verwendet werden, die sich zu Hypothesen formieren können [9]. Präzisere Hypothesen entstehen womöglich erst durch weitere Untersuchungen, durch die Verknüpfung mit Wissen und durch die Zusammenarbeit mit dem Patienten. Sie werden in einem weiteren Ausreifungsgrad gegen Ende des Untersuchungsprozesses bzw. der Befundaufnahme mit Aufstellen der physiotherapeutischen Diagnose formuliert [33-35].

\section{Schlussfolgerungen}

Durch die Befragung der acht physiotherapeutischen Experten der Ganganalyse konnte gezeigt werden, dass sich der Hypothesenbildungsprozess in der Physiotherapie dadurch auszeichnet, dass aufgrund von Beobachtungen Annahmen formuliert, geprüft, bestätigt oder verworfen werden, indem sie mit Bewertungen versehen und mit eigenem Wissen angereichert und verknüpft werden. Gebildete Hypothesen, welche in drei Typen (zu bewertende, zu interpretierende und zu bewertende und konditionale Annahme) gebildet werden, zeigen sich in allen Phasen des Beobachtungsprozesses der Ganganalyse und können in mehreren Iterationen gebildet werden. Die vorliegenden Ergebnisse zeigen deutlich, dass die Begriffe Hypothese und Hypothesenbildung derzeit in der Physiotherapie ungenau benutzt werden. Hypothesen ähneln in ihrer anfänglichen Stufe eher Heuristiken und bilden für Physiotherapeuten einen generalisierten Orientierungsrahmen, der weiteres Handeln bedingt und weitere Untersuchungsschritte einleitet. Erst, wenn Annahmen formuliert, geprüft, bewertet und somit gedanklich weiterverfolgt und entwickelt werden, können sie einen Hypothesenstatus erreichen. Somit wäre nicht jede Annahme, die angestellt, aber nicht weiterverarbeitet wird, als Hypothese, wie sie in der empirischen Forschung als wissenschaftliche Hypothese definiert wird, zu bezeichnen. Durch die aufgezeigten Schritte können aus Heuristiken präzisere Hypothesen, die gerichtete Zusammenhänge (z. B. Ursache-Folge-Beziehungen) beschreiben, gebildet werden.

Es ist zu vermuten, dass auch auf anderen Gebieten, in denen die beobachtende Bewegungsanalyse angewendet wird, ähnliche oder abgewandelte Formen von Hypothesen generiert werden. Dies muss durch weiterführende Arbeiten erforscht werden. Zur weiteren Einordnung und Beschreibung von Hypothesen sind überdies Studien notwendig, die sich mit der Theorie des Begriffes „Hypothese“ beschäftigen und Definitionen sowie Systematiken zur Hypothesenbildung in der Physiotherapie herausbilden. Studien können die Hypothesenbildung und Hypothesenkategorien im Interventionsprozess auch für verschiedene Patientengruppen fokussieren. Hier könnten die in der Forschungsarbeit gefundenen Typen und Merkmale unter unterschiedlichen Bedingungen untersucht und weiterentwickelt werden.

\section{Interessenkonflikt}

Die Autoren geben an, dass kein Interessenkonflikt besteht.

\section{Danksagung}

Die Autoren danken dem Labor für Bewegungswissenschaften der HAWK in Hildesheim sowie der FBL Functional Kinetics Gruppe für den Input und die angeregten Diskussionen.

\section{Literatur}

[1] Jones MA, Jensen GM, Edwards I. Clinical Reasoning in Physiotherapy. In: Higgs J, Jones MA, Loftus S, et al (Hrsg) Clinical reasoning in the health professions. Oxford: Butterworth Heinemann; 2008

[2] Edwards I, Jones M, Carr J et al. Clinical reasoning strategies in physical therapy. Physical Therapy 2004; 84: 312-330

[3] Doody C, McAteer M. Clinical Reasoning of Expert and Novice Physiotherapists in an Outpatient Orthopaedic Setting. Physiotherapy 2002; 88: 258-268

[4] Delitto A, Snyder-Mackler L. The Diagnostic Process: Examples in Orthopedic Physical Therapy. Physical Therapy 1995; 75: 203-211

[5] Jones M. Clinical reasoning and pain. Manual Therapy 1995; 1: 17-24

[6] Bartrow K, Osteopathie CP, Maassen A. Untersuchen und Befunden in der Physiotherapie. Untersuchungstechniken und Diagnoseinstrumente. Berlin: Springer; 2012

[7] Eberhard K. Einführung in die Erkenntnis- und Wissenschaftstheorie: Geschichte und Praxis der konkurrierenden Erkenntniswege. Stuttgart: Kohlhammer; 1999

[8] Flick U, von Kardorff E, Steinke I. Qualitative Forschung: Ein Handbuch. Reinbek: Rowohlt; 2005

[9] Bortz ], Döring N. Forschungsmethoden und Evaluation für Human- und Sozialwissenschaftler. Heidelberg: Springer; 2006 
[10] Häder M. Empirische Sozialforschung. Eine Einführung. Wiesbaden: VS Verlag für Sozialwissenschaften; 2010

[11] Diekmann A. Empirische Sozialforschung: Grundlagen, Methoden, Anwendungen. Reinbek: Rowohlt; 2007

[12] Flick U. Qualitative Sozialforschung: Eine Einführung. Reinbek: Rowohlt; 2007

[13] Creswell JW. Qualitative inquiry and research design: Choosing among five approaches. Thousand Oaks: Sage Publications; 2007

[14] Hoppe-Graff S, Engel I, Hoppe-Graff S et al. Psychologie. Berlin: Springer; 2003

[15] Zimbardo PG. Zimbardo Psychologie. Berlin: Springer; 1995

[16] Götz-Neumann K. Gehen verstehen: Ganganalyse in der Physiotherapie. Stuttgart: Thieme; 2016

[17] Martin K, Hoover D, Wagoner E et al. Development and reliability of an observational gait analysis tool for children with Down syndrome. Pediatric Physical Therapy 2009; 21: 261-268

[18] Toro B, Nester C, Farren P. A review of observational gait assessment in clinical practice. Physiotherapy theory and practice 2003; 19: 137-149

[19] Suppé B, Bongartz M. FBL Klein-Vogelbach Functional Kinetics praktisch angewandt: Gehen- Analyse und Intervention. Berlin: Springer; 2013

[20] Watelain E, Froger ], Barbier F et al. Comparison of clinical gait analysis strategies by French neurologists, physiatrists and physiotherapists. Journal of rehabilitation medicine 2003 ; 35: 8-14

[21] Van Someren MW, Barnard YF, Sandberg JAC. The think aloud method: a practical approach to modelling cognitive processes. London: Academic Press; 1994

[22] Ericsson KA, Simon HA. Verbal reports as data. Psychological review 1980; 87: 215
[23] Embrey DG, Guthrie MR, White OR et al. Clinical decision making by experienced and inexperienced pediatric physical therapists for children with diplegic cerebral palsy. Physical Therapy 1996; 76: 20-33

[24] Gilliland S, Wainwright SF. Patterns of Clinical Reasoning in Physical Therapist Students. Physical therapy 2017; 97: 499-511

[25] Langridge N, Roberts L, Pope C. The clinical reasoning processes of extended scope physiotherapists assessing patients with low back pain. Manual therapy 2015; 20: 745-750

[26] Perry J. Gait Analysis, Normal and Pathological Function. Monterey: Slack; 1992

[27] Suppé B, Bongartz M. FBL Klein-Vogelbach Functional Kinetics praktisch angewandt: Gehen - Analyse und Intervention. Berlin: Springer; 2013

[28] Fonteyn ME, Kuipers B, Grobe S]. A description of think aloud method and protocol analysis. Qualitative health research 1993; 3: 430-441

[29] Strauss A, Corbin J. Grounded theory. Grundlagen qualitativer Sozialforschung. Weinheim: Beltz; 1996

[30] Glaser BG, Strauss AL. Grounded theory: Strategien qualitativer Forschung. Berlin: Huber; 2010

[31] Polanyi M. Implizites Wissen. Frankfurt/M.: Suhrkamp; 2016

[32] Klemme B, Siegmann G. Clinical Reasoning: Therapeutische Denkprozesse lernen. Stuttgart: Thieme; 2015

[33] Trocha M, Aigner A-K, Brandt $\mathrm{H}$ et al. Befundest du noch - oder diagnostizierst du schon? physioscience 2014; 10: 24-28

[34] Hengeveld E. Untersuchen als Prozess, Clinical Reasoning. In: HüterBecker A, Dölken M, (Hrsg) Untersuchen in der Physiotherapie. Stuttgart: Thieme; 2011

[35] World Confederation for Physical Therapy. Policy statement: Description of physical therapy. 2017 www.wcpt.org/policy/ps-descriptionPT (04.12.2019) 